\title{
Enhancing Independent Time-Management Skills of Individuals With Mental Retardation Using a Palmtop Personal Computer
}

\author{
Daniel K. Davies, Steven E. Stock, and Michael L. Wehmeyer
}

\begin{abstract}
Achieving greater independence for individuals with mental retardation depends upon the acquisition of several key skills, including time-management and scheduling skills. The ability to perform tasks according to a schedule is essential to domains like independent living and employment. The use of a portable schedule prompting system to increase independence and self-regulation in time-management for individuals with mental retardation was examined. Twelve people with mental retardation participated in a comparison of their use of the technology system to perform tasks on a schedule with use of a written schedule. Results demonstrated the utility of a Palmtop computer with schedule prompting software to increase independence in the performance of vocational and daily living tasks by individuals with mental retardation.
\end{abstract}

Achieving greater independence for individuals with mental retardation depends upon the acquisition of a variety of important skills in a wide array of life domains, including such areas as work, transportation, cooking, and personal care. Achieving enhanced independence across these diverse areas has a common denominator-the need to learn to manage time and maintain and manage a personal schedule. For example, using the public transportation system successfully requires that a person adhere to a series of timemanagement and personal scheduling activities, including identifying what time to get ready in order to catch the bus, what time to leave home or work to get to the bus stop, and what time the bus is scheduled to arrive. Similarly, many work-related tasks rely on the capacity of the worker to allot his or her time appropriately between and among multiple tasks (Martin, EliasBurger, \& Mithaug, 1987). Many people with mental retardation have less success in time-management and personal scheduling activities because of limited memory skills and difficulty with the concepts of time and the passage of time. These memory and timemanagement limitations, in turn, contribute to reduced independence in many major life domains.

Despite the potential imp ortance of time -man- agement and personal scheduling skills for people with mental retardation, there has been only limited research on promoting and enhancing these skills. The general psychology and education literature includes numerous reports about the importance of time-management skills for all people. For example, several researchers have linked time-management skills with successful college performance, including higher achievement scores and greater maturity (Britton \& Tesser, 1991; Trueman \& Hartley, 1996). There have been fewer examinations of issues of time-management with students who have disabilities. Manganello (1994) stressed the importance of such skills for older students with learning disabilities and provided strategies, including use of a time-management $\log$, to promote better skills. Sowers, Rusch, Connis, and Cummings (1980) examined the impact of training in time-management skills with three adults who had mental retardation and basic time-telling abilities, finding that these individuals could be taught to engage in independent time-management. The exception to this relative lack of attention has been in the area of teaching self-scheduling as a self-management skill. There are numerous studies showing that people with mental retardation can learn self-scheduling skills (Bambera \& Ager, 1992; Lovett \& Haring, 1989; 
Martin, Elias-Burger, \& Mithaug, 1987). In many of these self-management studies, investigators used antecedent cue regulation and permanent antecedent prompts (including picture books and other visual aides) to promote adherence to a schedule.

The widespread growth and use of technology by most people to assist in routine time-management and scheduling activities constitutes another means of offering visual (and audio) aides to schedule adherence and introduces a means to mitigate the negative impact of cognitive limitations on timemanagement and scheduling tasks for people with mental retardation. From personal information managers and personal computer-based calendars to Palm Pilot devices and palmtop personal computers, there are a wide array of computer-based programs and devices that provide mechanisms to effectively manage one's time and to schedule activities and tasks. Unfortunately, most of these software programs and devices are too complex for people with mental retardation to operate independently, a fre quent problem with technological and assistive devices (Davies, Stock, \& Wehmeyer, 2001; Wehmeyer, 1998a). That is not to say, however, that such software supports and devices cannot be designed using principles of universal design that would make them more accessible for people with mental retardation. In fact, the emergence of palmtop personal computers, such as those utilizing the Windows CE operating system, provides a promis ing platform from which to design supports related to time-management and scheduling. These devices are portable, involve the use of a touch screen for operation, can utilize audio and visual output, and are relatively affordable. In addition, like its larger cousin, the desktop PC, custom software can be written and loaded onto palmtop PCs.

There are two basic elements in task performances: what to do and when to do it. The "what to do" element involves knowledge about the steps of a particular skill, such as taking a shower or bussing tables at a restaurant. We have demon-strated, in a separate study, the efficacy of using appropriately designed software on a palmtop computer to provide supports to individuals with mental retardation with the "what to do" com-ponent of task performance on vocationally oriented tasks (Davies, Stock, \& Wehmeyer, 2002). In the present study we address the "when to do it" element of task performance, which relates to following a schedule of when to perform tasks or activities. Not only is the "when to do it" element often an area of dependency for people who cannot tell time, but these same people often have difficulty reading directions, which can also result in dependence in the "what to do" part of time-management.

Given the emphasis in the last decade on the importance of promoting self-determination (Wehmeyer, 1992, 1998b, 2001), it is important to consider the implications of problems in time-management and personal scheduling not only on a person's relative independence, but also on his or her selfdetermination and perceptions of control. Mithaug (1998) noted that self-determination always has a social context; that is, it is always self- versus other determination. When people are unable to adhere to schedules and manage time segments, they often become dependent upon others to prompt them or to manage their affairs, a circum stance that will limit a person's opportunities to experience control and self-direction and, ultimately, likely contribute to a person's belief that he or she cannot control his or her affairs.

Self-management skills are important for individuals with mental retardation to exert greater control in their lives (Agran, 1997; Wehmeyer, Agran, \& Hughes, 1998); enabling them to manage their own schedules both removes dependency on others and creates opportunities for control. There is considerable evidence that people with mental retardation can, indeed, self-manage their behavior (Martin, Burger, Elias-Burger, \& Mithaug, 1988). For example, individuals with mental retardation have been taught to use self-instruction to solve a variety of work problems (Agran \& Moore, 1994; Hughes, 1992; Hughes \& Rusch, 1989); to complete complex, multi-step sequences (Agran, Fodor-Davis, \& Moore, 1986); and to generalize desired responding across changing work environments (Agran \& Moore, 1994) as well as increase community access (Hughes \& Agran, 1994). Similarly, individuals with mental retardation have used self-monitoring procedures for facilitating job-task changes (Sowers, Verdi, Bourbeau, \& Sheehan, 1985) and evaluating how often a task was completed (Mace, Shapiro, West, Campbell, \& Altman, 1986). Time-management and scheduling skills serve an "executive function" in managing one's own behavior by enabling the person to control when he or she engages in self-managed or self-directed tasks.

Our purpose in this study was to examine the efficacy of the Schedule Assistant software package and the use of a palmtop PC for enhancing personal 
and the use of a palmtop PC for enhancing personal schedule maintenance and time-management skills for individuals with mental retardation. This analysis addressed three primary questions: (a) Can an automated schedule reminder system provide sufficient cues to help individuals with mental retardation react to time-based auditory prompts? (b) Can auditory prompts generated from an automated schedule reminder system provide adequate information for individuals with mental retardation to follow instructions? (c) Can an automated schedule reminder system reduce the number of prompts required from external support persons to help keep people with mental retardation on a schedule?

\section{Method}

\section{Participants}

Participants were 12 volunteers ( 8 men, 4 women) receiving community-based vocational supports from a local agency providing services to adults with mental retardation and through a school district's community-based program for students with mental retardation ages 18 to 21 . There were 12 participants ( 8 men, 4 four women) in the study group. They ranged in age from 19 to 46 years (mean age $=34.17$ ). Scores from intelligence testing (the Wechsler Adult Intelligence Scale-Revised-WAISR) for participants ranged from 45 to 90 (mean IQ =62.6). All individuals except one had IQs below 70. The other individual included in the study had a developmental disability (IQ = 90) and was a participant in a high school special education transition program. Informed consent was obtained from all participants prior to beginning the study, and they were compensated for their participation.

\section{Device Design}

Schedule Assistant is an automated multimedia scheduling system designed to operate on the Windows CE palmtop computer platform that assists a special needs user in maintaining a personal schedule by providing visual and audio prompts according to a schedule of events. Audio messages and digital pictures can be created to provide cues to the person to begin performing scheduled activities, such as getting ready to catch the bus or making a phone call at a prescribed time. Options are provided to replay the message automatically or, if requested by the user, to require confirmation that the activity has been completed by the user. The device has an internal speaker to play audio messages; headphones or an earphone can also be used. Palmtop computers are portable, unobtrusive devices and are in creasingly employed by the general public, thus making them desirable for use in the community. In fact, use of mainstream technology devices to provide supports has the effect of including as opposed to excluding individuals with mental retardation in society due to the presence of potentially stigmatizing special equipment.

\section{Setting}

Because schedule time constraints for this study (6 months from system design to evaluation) ruled out the opportunity for extended evaluation in applied contexts, we designed a testing environment that represented both vocational and residential tasks common to those settings. A room was provided by a small local software company (approxi mately 12' by $16^{\prime}$ ) that was typically used as a product staging area and break room. It included a large work table, one window with a view to the outdoors, several file cabinets and book shelves, and a number of shipping boxes stacked along a wall. In addition, the room contained a television/VCR, microwave, and coffee pot in a corner of the room used as a break area. Study materials were placed on the work table. Five of the required tasks were performed by the participant while seated at the work table, and three (Tasks 5, 6, and 8 , see below) required the participants to get up to do a task that was clearly within view of the table. No people other than researchers and participants were present in the room during training and testing.

\section{Procedure}

Each participant attempted to complete an eight-item schedule using both the Schedule Assis tant system and a traditional written schedule. The first four tasks were vocationally focused, with the remaining four tasks common to residential settings. The tasks were designed to be simple motor tasks because our intent was to measure people's ability to initiate a relatively known task in a timely manner, not the ability to learn new tasks. Therefore, even if a participant could not read a written prompt, if he or she asked for help within a minute after the scheduled time, they were given credit for initiating the task. Prior to beginning a test session, participants were familiarized with each task. Familiarization included a demonstration of each task followed by the opportunity for test subjects to successfully perform the task. Errors in specific task 
Time-management and personal scheduling

task performance (e.g., crooked label or spilled water) were not counted against subjects. The focus of the as sessment was on the ability to initiate tasks at the proper time, not the ability to perform the tasks without error. These eight tasks were to (a) begin labeling floppy disks, (b) stop and take a break, (c) resume labeling disks, (d) stop and put completed disks in the box, (e) turn off the coffee pot, (f) close the drawer on the file cabinet, $(g)$ water the plant, and (h) go out into the hallway and inform the researcher you are done.

The Schedule Assistant system was set up with a schedule prompting the users to complete these eight tasks over a 25 -minute period. They received training from researchers on how to use the portable computer system until they were able to independently respond to the system's alarm on consecutive trials. An informed consent statement was reviewed with each person in understandable terms, and participants were assured that they could ask for help, ask other questions, or discontinue at any time during the session.

A large print, bold-typed task list was developed with simple language providing the same eight task prompts listed previously. The times for each task to be performed in a given testing session were obtained from either Set A or Set B (see discussion below) in the Schedule Assistant system to provide consistency between the two approaches, and these times were then hand written on the form next to the respective task in large block letters. A large display digital clock was placed on the table directly in front of each participant. They again received training from researchers on how to use the traditional system until they were able to independently respond to a designated time displayed on the digital clock on consecutive trials. In both testing situations, participants were allowed to select a videotape to watch to (a) fill in gaps between the completion of scheduled activities and the time to engage in the next task and (b) serve as a mild distraction throughout the evaluation sessions. This distraction was provided to simulate the real-world distractions that are often present through the course of performing various activities. The volume on the VCR was kept relatively low.

Two additional control procedures were added to the experimental sessions. First, order of presentation of the two schedule-maintenance approaches was randomized (i.e., the Schedule Assistant system vs. the traditional prompting system). In addition, the eight
D.K. Davies, S.E. Stock, and M.L. Wehmeyer

tasks were presented randomly using two different time sets. That is, a Set A and Set B were developed using the same tasks but with differences in the amount of time between tasks. Each participant's session lasted approximately one hour.

The evaluation took place over a 4 week period. There was one independent variable with two levels; the traditional prompting method and the Schedule Assistant automated time-management system. The dependent variables were schedule accuracy as measured by the ability to change actions on time and assistance required as measured by prompts required to complete a task.

\section{Research Design and Analysis}

We utilized a two-group within-subjects design (Campbell \& Stanley, 1963). Each participant com pleted a series of eight simulated vocational and residential tasks twice, once using the' Schedule Assis tant system and once using a written schedule of instructions. Written instructions were provided with simple wording in large print along with the associated time that each task was to be performed. Participants selected a videotape to play on a VCR in the room to serve as a mild distraction and occupy their time during breaks in activity during the sessions. Each participant received training on the eight tasks as well as on how to use the two schedule prompting systems.

While the participants engaged in the evaluation sessions, data were collected on each of the dependent measures. Each task was marked as completed if it was initiated within one minute after the prescribed time. The number of times prompts were required, if any, to complete a task was also recorded. A prompt was recorded if the person asked for help related to the time at which a task was to be completed or asked what the task was that needed to be completed. However, no prompt was recorded if participants asked a question relating only to the accuracy or quality of the task being performed (e.g., Is the label on straight enough?). A prompt was also recorded if the participant did not take the scheduled break in Task 2, as initiation of Task 3 (i.e., resuming the disk-labeling activity) was dependent upon completion of Task 2 . In addition to these quantitative assessments, an interview format was used to collect social validation data. from both the participants and their support staff members regarding the utility of the automated time-management approach of the Schedule Assistant. These data were collected to aid in the 
interpretation of the project results. Given the small sample size, we utilized a within-subjects design to increase the power of our test for mean differences. Sandler's A statistic (a form of $t$ test derived from Student's $t$ ratio appropriate for use with correlated samples) was selected given the correlated score samples in our test and the need to investigate mean differences between the two schedule-maintenance systems. Mean differences in number of help prompts required and the number of schedule errors made were tested for significance at the .05 level with a one-directional test.

\section{Results}

Data analysis using paired comparisons $t$ tests yielded significant differences for both dependent variables between the two schedule-maintenance approaches. Participants required significantly less assistance $(\mathrm{M}=1.33$ prompts, standard deviation [SD] $=2.27$ ) than when following the written schedule $(\mathrm{M}=4.58$ prompts, $\mathrm{SD}=3.50)$ when using the Schedule Assistant system, $t(11)=3.59$, p $<.002$. In addition, individuals using the Schedule Assistant prototype made significantly fewer schedule errors $(\mathrm{M}=.67, \mathrm{SD}=2.31)$ than when using the written schedule $(\mathrm{M}=3.58, \mathrm{SD}=2.39)$, $t(11)=4.70, \mathrm{p}<.001$. In fact, all participants except one were able to complete all required tasks on time when using the Schedule Assistant prototype, whereas only one individual was able to complete the set of tasks on time when using the written reminder system.

\section{Discussion}

The results of this study support the hypothesis that using an automated schedule prompting system could increase the ability of individuals with mental retardation to initiate scheduled activities independently and at appropriate times. Furthermore, these findings extend the limited information about the capacity of people with mental retardation to engage in time-management activities, a potentially important aspect of self-management. Participants both reported and demonstrated their satisfaction with their success in using the system and their preference for using Schedule Assistant as opposed to a written schedule approach. Several examples of statements made by participants in expressing their satisfaction and appreciation of the system in cluded:
I could useoneof these.

Because it helps my memory.

I wish I had one of these to catch the bus.

Helps me out soI won't forget.

That's prettycool.

Oh, I'mso good at this -I got it!

It helps me by doing a greatjob.

It would tell me when it's time to go.

Most participants were able to name situations in their lives where the Schedule Assistant system would be useful, such as when to catch the bus, take a break at work, or when to take their medications. School and agency support staff members who observed evaluation sessions also noted the likely benefits of the system and were able to describe situations where they felt individuals they supported could use Schedule Assistant (e.g., to guide them through the tasks and events at their places of work, for completion of morning routines).

Interpretation of these data is, necessarily, preliminary given several factors. Because of the small sample size, one must be cautious of even large differences between means, and these data must be interpreted with this in mind. Second, because the pilot testing of the computerized scheduling system had to be completed in a short time span due to funding requirements ( 6 months from design to evaluation), it was not feasible to provide participants with more exposures to the two procedures. In addition, for similar reasons, evaluations were conducted within the context of the research firm that developed the device, and before generalizations can be made about the potential of devices such as the Schedule Assistant to benefit people with mental retardation, research will be needed with a larger sample who use the device in ecologically valid settings, such real work settings or in their home. The demands of the device-development process limited our capacity to involve more students or to expand the evaluation to community settings, but we contend that there is value to this study for two reasons. First, this is a newly emerging technology, and it is important to evaluate the potential for its use with people who have mental retardation early in the evolution of such products so 
that we can identify limitations and barriers in time to influence future developments. It is important that the handheld computers and the software running on these devices adhere to universal design principles, including the need to be accessible for people with mental retardation and other cognitive disabilities. Through evaluations such as this one, we can better advocate for changes in hardware and software that would benefit people with mental retardation. Second, although the evaluations were not conducted in work or living situations, there is no compelling reason to believe that the device would be any less effective in those settings.

The benefits from the utilization of the Schedule Assistant can most likely be attributed to the combination of the use of a multimedia software development approach and the automated timing technology available in palmtop computers. The recorded audio messages provided by the Schedule Assistant system provided the necessary format for all participants to understand exactly what task was to be performed. The only instances of participants needing prompting while using the Schedule Assis tant system were when asking for reassurance or direction on how to use the system; specifically, to remind them to tap the screen with their finger when hearing the alarm that automatically played at the prescribed time. This generally occurred only during the first few scheduled activities, while test participants were still becoming comfortable with

Conversely, the capacity of the system to assist participants to perform tasks independently and on time demonstrated the strongest support for Schedule Assistant. Only one participant was unable to complete all tasks on time when using Schedule Assistant. However, her support person also indicated a belief in her potential for eventually being able to use the system given extended practice and using her mother's voice as the source of the recorded audio reminders.

For all other participants, there were no instances of late or early performance of tasks, largely, we suspect, because of the simple prompting interface of-the combined system - a generated "ding" followed by the simple task of pressing the touch screen to hear the recorded audio cue. A few individuals sought reassurance during the first one or two trials of these sequences, but inevitably all of them indicated their confidence at being able to complete scheduled tasks on time when using the Schedule Assistant.

We found that when using the Schedule As sistant, participants made significantly fewer errors in performing tasks according to a schedule than when not using the system, even though the num- using the system.

The other dependent variable in the study was the ability of participants to perform tasks on time. In both the Schedule Assistant and the traditional written schedule approach, tasks were marked as completed on time if the person either completed the task or asked for help in completing (or understanding) the task within one minute after the scheduled time. Therefore, even if a participant could not read a written prompt, if he or she asked for help within a minute after the scheduled time, a "yes" was recorded. A "no" was only recorded if a participant neither initiated a task nor asked for help within one minute after the scheduled time.

When using the traditional written schedule system, participants often failed to perform tasks on time for two reasons. First, in some cases they became distracted or otherwise did not pay close enough attention to the time to perform the task as scheduled. Second, some individuals with more severe cognitive disabilities were unable to correctly relate the written time with the time displayed on the digital clock. For instance, one participant was able to correctly identify some of the times, but had difficulty discriminating between the numbers 2 and 5 when they were displayed. In another case, a participant performed the last four tasks consecutively whenever the digital clock changed to the next minute, disregarding the written schedule completely.

ber of requests they made for help were fewer. This finding provides support for the contention that a portable palmtop computer system can be a useful adjunct to personal schedule maintenance and time-management for individuals with mental retardation. One obvious benefit resulting from using portable multimedia technology to enhance independent schedule management is to reduce the staff time required to ensure that individuals adhere to a work schedule. Individuals who could benefit from the self-directed, self-prompting nature of the device would be able to learn to accomplish tasks on time by using the Schedule Assistant system and would require less external prompting from job coaches or supported living staff. This would enable support professionals to focus more attention on people who require additional intensive supervision and training. Moreover, we hypothesize that the increased self-direction provided by the process will enhance individuals' self-determination. It is worth noting, however, that there is some "up front" time needed for support personnel to set up the handheld device and personalize it as well as time related to maintenance and care. The system has been designed to be user-friendly and to limit the time 
needed to set it up. Nonetheless, use of devices such as this do not mean that support personnel are not needed at all.

Handheld or palmtop PCs are becoming increasingly prevalent in society. They present a seemingly ideal platform in which to design soft ware that can provide supports to people with mental retardation in the community. Because they are commercially available (not to mention socially desirable), there is no stigmatizing effect from their use. As they become more widely used by the general population, the cost of owning one will undoubtedly decrease, making them more economi cally viable. Even at their current prices (ranging from $\$ 300$ to $\$ 600$ ), they tend to be more economical than other types of specially designed technology. They are, as we have indicated, highly portable, lend themselves to use in training and support situations, and employ a simple input process (touch screen). There are, however, some barriers to their wide utilization by people with mental re tardation that we have identified. Those barriers are similar to those that have limited the use of technology by people with mental retardation over time (Wehmeyer, 1998a), including the relative userfriendliness (or lack thereof) of the operating system and the device itself (e.g., switch placement and ease of use). Moreover, the development of software that is accessible to people with mental retardation remains a problem area. In all, however, and despite these limitations, we suggest that handheld computers with appropriately designed software present a potentially powerful way to decrease the dependence of people with mental retardation on others for training and support and to increase selfdetermination and community inclusion.

\section{References}

Agran, M. (1997). Student-directed learning: Teaching self-determination skills. Pacific Grove, CA: Brooks/Cole.

Agran, M., Fodor-Davis, J., \& Moore, S. (1986). The effects of self-instructional training on job-task sequencing: Suggesting a problem-solving strategy. Education and Training in Mental Retardation, 21, 273-281.

Agran, M., Fodor-Davis, J., Moore, S. C., \& Martella, R. C. (1992). The effects of peer-deliv ered self-instructional training on a lunch-making work task for students with severe handicaps. Education and Training in Mental Retardation, 27, 230-240.
Agran, M., \& Moore, S. C. (1994). How to teach selfinstruction of job skills. Washington, DC: American Association on Mental Retardation.

Bambera, L. M., \& Ager, C. (1992). Using selfscheduling to promote self-directed leisure activity in home and community settings. Journal of the Association for Persons with Severe Handicaps, 17, 67-76.

Britton, B. K., \& Tesser, A. (1991). Effects of timemanagement practices on college grades. Journal of Educational Psychology, 83, 405-410.

Campbell, D. T., \& Stanley, J. C. (1963). Experimental and quasi-experimental designs for research. Boston: Houghton Mifflin.

Davies, D. K., Stock, S. E., \& Wehmeyer, M. L. (2001). Enhancing independent internet access for individuals with mental retardation through use of a specialized web browser: A pilot study. Education and Tra ining in Mental Retardation and Developmental Disabilities, 36, 107-113.

Davies, D. K., Stock, S. E., \& Wehmeyer, M. L. (2002). Enhancing independent task performance for individuals with mental retardation through the use of a handheld self-directed visual and audio prompting system. Education and Training in Mental Retardation and Developmental Disabilities, 37, 209-218.

Hughes, C. (1992). Teaching self-instruction utilizing multiple exemplars to produce generalized problem-solving by individuals with severe mental retardation. American Journal on Mental Retardation, 97, 302-314.

Hughes, C., \& Agran, M. (1994). Teaching persons with severe disabilities to use self-instruction in community settings. Journal of the Association for Persons with Severe Handicaps, 18, 261-274.

Hughes, C., \& Rusch, F R. (1989). Teaching supported employees with mental retardation to solve problems. Journal of Applied Behavior Analysis, 22, 365-372.

Lovett, D. L., \& Haring, K. A. (1989). The effects of self-management training on the daily living of adults with mental retardation. Education and Training in Mental Retardation, 24, 306-323.

Mace, F. C., Shapiro, E. S., West, B. J., Campbell, C., \& Altman, J. (1986). The role of reinforcement in reactive self-monitoring. Applied Research in Mental Retardation, 7, 315-327.

Manganello, R. E. (1994). Time-management instruction for older students with learning disabilities. Teaching Exceptional Children, 26(2), 60-62. 
Martin, J. E., Burger, D. L., Elias-Burger, S., \& Mithaug, D. E. (1988). Applications of selfcontrol strategies to facilitate independence in vocational and instructional settings. In $\mathrm{N}$. Bray (Ed.), International review of research in mental retardation (pp. 155-193). San Diego: Academic Press.

Martin, J. E., Elias-Burger, S., \& Mithaug, D. E. (1987). Acquisition and maintenance of time-based task change sequence. Education and Training in Mental Retardation, 22, 250255.

Mithaug, D. (1998). Your right, my obligation? Journal of the Association for Persons with Severe Disabilities, 23, 41-43.

Sowers, J., Rusch, F, Connis, R. T., \& Cummings, L. E. (1980). Teaching mentally retarded adults to time-manage in a vocational setting. Journal of Applied Behavior Analysis, 13, 110-128.

Sowers, J., Verdi, M., Bourbeau, P., \& Sheehan, M. (1985). Teaching job independence and flexibility to mentally retarded students through the use of a self-control package. Journal of Applied Behavior Analysis, 18, 8185.

Trueman, M., \& Hartley, J. (1996). A comparison between the time-management skills and academic performance of mature and traditional-entry university students. Higher Education, 32, 199-215.

Wehmeyer, M. L. (1992). Self-determination and the education of students with mental retardation. Education and Training in Mental Retardation, 27, 302-314.

Wehmeyer, M. L. (1998a). National survey of the use of assistive technology by adults with mental retardation. Mental Retardation, 36, 44-51.

Wehmeyer, M. L. (1998b). Self-determination and individuals with significant disabilities: Examining meanings and misinterpretations. Journal of the Association for Persons with Severe Handicaps, 23, 5-16.
Wehmeyer, M. L. (2001). Self-determination and mental retardation. In L.M. Glidden (Ed.), International review of research in mental retardation (Vol. 24, pp. 1-48). Hillsdale, NJ: Erlbaum.

Wehmeyer, M. L., Agran, M., \&. Hughes, C. (1998). Teaching self-determination to youth with disabilities: Basic skills for successful transition. Baltimore: Brookes.

Received 4/9/01, first decision 5/18/01, accepted 9/7/01.

Editor-in-charge: Steven J. Taylor

This article is based upon work supported by the U.S. Department of Education under PO ED-99-PO-4884. Any opinions, findings, conclusions, or recommendations expressed are those of the authors and do not necessarily reflect the views or policies of the Department of Education. The opportunity to interview support professionals was critical to the success of this project. The researchers thank the staff at Colorado Springs School District 11 and Cheyenne Village, Inc., who provided opinions, feedback, and otherwise facilitated various tasks in the project. In addition, we thank those individuals who volunteered to participate in the Schedule Assistant study and other testing activities conducted as part of this project. Without their help, the project could not have been accomplished.

\section{Authors:}

Daniel K. Davies, MA, President and Founder (E-mail: dan@ablelinktech.com), and Steven E. Stock, MA, Vice-President, AbleLink Technologies, Inc., 528 N. Tejon St., Suite 100, Colorado Springs, CO 80903. Michael L. Wehmeyer, PhD, Associate Professor, Beach Center on Disability and Kansas University Center on Developmental Disabilities, University of Kansas, 1200 Sunnyside Avenue, Room 3136, Lawrence, KS 66045. 
